\title{
Giant resonant enhancement of optical binding of dielectric disks
}

\author{
E.N. Bulgakov, ${ }^{1}$ K.N. Pichugin, ${ }^{1}$ and A. F. Sadreev ${ }^{1,}$ a) \\ Kirensky Institute of Physics, Federal Research Center KSC SB RAS, 660036 Krasnoyarsk, \\ Russia
}

(Dated: 20 July 2020)

Two-parametric variation over the aspect ratio of each disk and distance between disks gives rise to numerous events of avoided crossing of resonances of individual disks. For these events the hybridized anti-bonding resonant modes can acquire a morphology close to the Mie resonant mode with high orbital momentum of equivalent sphere. The $Q$ factor of such resonance can exceed the $Q$ factor of isolated disk by two orders in magnitude. We show that dual incoherent counter propagating coaxial Bessel beams with power $1 \mathrm{~mW} / \mu \mathrm{m}^{2}$ with frequency resonant to such a antibonding modes result in unprecedented optical binding forces up to decades of nano Newtons for silicon micron size disks. We show also that a magnitude and sign of optical forces strongly depend on the longitudinal wave vector of the Bessel beams.

\section{INTRODUCTION}

The response of a microscopic dielectric object to a light field can profoundly affect its motion. A classical example of this influence is an optical trap, which can hold a particle in a tightly focused light beam $\frac{1}{*}$. When two or more particles are present, the multiple scattering between the objects can, under certain conditions, lead to optically bound states. This is often referred to peculiar manifestation of optical forces as optical binding (OB), and it was first discovered by Burns et al. on a system of two plastic spheres in water in $1989^{2}$. Depending on the particle separation, OB leads to attractive or repulsive forces between the particles and, thus, contributes to the formation of stable configurations of particles. The phenomenon of OB can be realized, for example, in dual incoherent counter propagating beam configurations $\mathrm{s}^{3-9}$. Many researchers have analyzed $\mathrm{OB}$ force quantitatively in theory. Chaumet et $a l^{10}$ and $\mathrm{Ng}$ et $a l^{11}$ calculated the OB force under illumination of two counter propagating plane waves. Čižmár et $\mathrm{al}^{12}$ presented the first theoretical and experimental study of dielectric sub-micron particle behavior and their binding in an optical field generated by interference of two counter propagating Bessel beams.

An excitation of the resonant modes with high $Q$ factor in dielectric structures results in large enhancement of near electromagnetic (EM) fields and respectively in extremely large EM forces proportional to squared EM fields. First, sharp features in the force spectrum, causing mutual attraction or repulsion between successive photonic crystal layers of dielectric spheres under illumination of plane wave has been considered by Antonoyiannakis and Pendry 13 . Because of periodicity of structure each layer is specified by extremely narrow resonances which transform into the sharp resonant bonding and anti-boding resonances for close approaching of the layers. Also it was revealed that the lower frequency bonding resonance forces act to push the two layers together and the higher frequency anti-bonding resonance to pull them apart. Later these disclosures we reported for coupled photonic crystal slabs ${ }^{14}$, for coupled asymmetric membranes ${ }^{15}$, and two

\footnotetext{
${ }^{a)}$ Electronic mail: almas@tnp.krasn.ru.
}

planar dielectric photonic metamaterials 16 due to existence of resonant states with infinite $Q$ factor (bound states in the continuum).

Even two particles can demonstrate precedents of extremely high $Q$ factors resonant modes owing to the mechanism of avoided crossing. The bright example is avoided crossing of whispering-gallery modes (WGM) in coupled microdisks ${ }^{17}$ which resulted in extremely high enhancement of OB between coupled WGM spherical resonators 18 . However the WGM modes can be excited only in spheres with large radii of order $30 \mu \mathrm{m}$. Respectively, the OB force for such massive particles can be turned out not so significant. In the present letter we offer a solution to the problem by use of two coaxial silicon disks of micron sizes shown in Fig. 1 which show extremely high- $Q$ factors in the subwavelength regime. Owing to two-parametric (over the aspect ratio and distance between disks) avoided crossing of low order resonances the anti-bonding resonant mode acquires a morphology of the high order Mie resonant mode of effective larger sphere with extremely small radiation losses 19 . We show also that a magnitude and what is more interesting the sign of the OB force strongly depend on the wave number of the Bessel beams that opens additional options to manipulate high index particles optically.

\section{TWO-PARAMETRIC AVOIDED CROSSING OF RESONANCES}

The phenomenon of avoided resonance crossing has attracted interest in photonics by the enhancement of the quality factor of resonant modes in coupled optical microcavities in the WGM regime ${ }^{20.21}$. An interest is renewed when high $Q$ resonant modes were revealed even in isolated dielectric disk show high $Q$ factor owing to avoided crossing of resonant modes for variation of aspect ratio in subwavelength range ${ }^{22}$. Because of importance of this result we reproduce that process in Fig. 2(a). At the aspect ratio $a / h=0.71$ the $Q$ factor reaches maximal value around 160 for high index optical material ( $\mathrm{Si}$ ) as shown in Fig. 2(b). One can see that the hybridized mode has a morphology very close to the morphology of the Mie resonant mode with orbital momentum $l=3$ in equivalent sphere whose volume equals $\pi a^{2} h$. This mode 


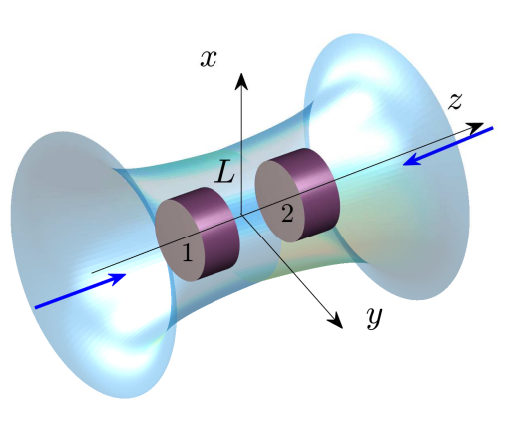

FIG. 1. Two silicon disks with the radius $a$, permittivity $\varepsilon=15$ are illuminated by dual counter-propagating mutually incoherent Bessel beams with zero azimuthal index $m=0$. Light intensity of each beam $1 \mathrm{~mW} / \mu \mathrm{m}^{2}$.
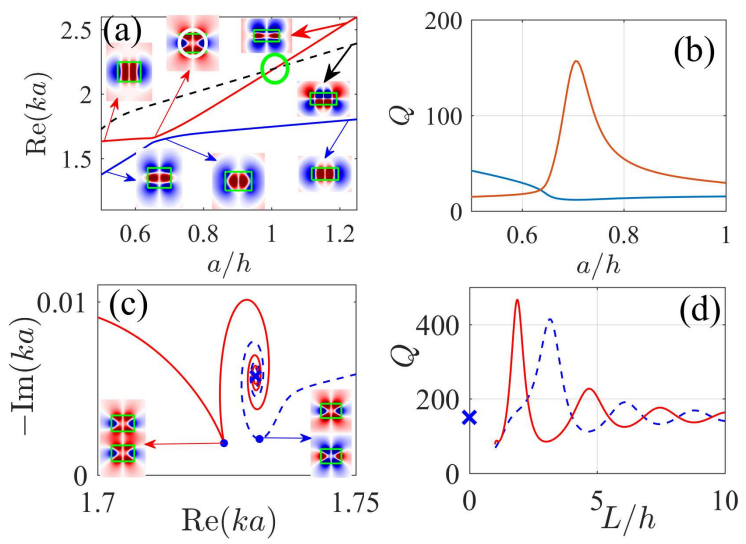

FIG. 2. (a) Avoided crossing of two TE resonances whose modes are symmetric relative to $z \rightarrow-z$ and (b) their $Q$ factors versus the aspect ratio $a / h$ in isolated silicon disk with dielectric constant $\varepsilon=12$. Insets show the profiles of tangential component of electric field $E_{\phi}$. Crosses mark the degenerate resonant frequencies and respectively the $Q$ factor of isolated disks. (c) and (d) Evolution of resonances and the $Q$ factor vs distance between disks at $a / h=0.71$.

highlighted by white circle in upper inset of Fig. 2 (a). The spherical particle has the minimal surface compared to given volume and therefore smallest radiation losses compared to particles with another shapes. That explains a reason to enhance the $Q$ factor by one order compared to the disk. That explains the peak in the $Q$ factor of the corresponding resonant mode in Fig. 2(b).

If to fix this optimal aspect ratio 0.71 and traverse over the distance between disks the $Q$ factor enhances by a few times compared to the isolated disk ${ }^{23}$. Evolution of the resonances and the behavior of the quality factor $Q=-\frac{\operatorname{Re}(k a)}{2 \operatorname{Im}(k a)}$ are shown in Fig. 2 (c) and (d) respectively. At $L \gg a$ the resonances are degenerate that is marked by cross in Fig. 2 (c). For an approaching of disks the resonances are split and evolve spirally so that at some distances the imaginary parts of hybridized complex resonant frequencies reach some minima marked in Fig. 22 (c) by closed circles. The spiral behavior of complex resonant frequencies is a result of radiation of leaky resonant modes by one disk and consequent scattering by the another. These scattering processes give rise to the coupling $e^{i k L} / L^{2}$ which hybridizes the resonant modes of separate disks as the leaky bonding and anti-bonding resonant modes 23 . They are shown in insets of Fig. 2 (c) at left (bonding mode) and right (anti-bonding mode) at those distances at which the $Q$ factor reaches the maxima, $L / a=1.87$ and $L / a=3.16$ respectively as shown in Fig. 22(d).

\section{OPTICAL BINDING FORCES}

It is clear that the same mechanism of consequent scattering processes underlines the optical binding stimulated by incident Bessel beam and respectively the OB force. It is reasonable to consider the OB force at the optimized aspect ratio $a / h=0.71$ where the $Q$ factor of the bonding and antibonding resonant modes show the maximal $Q$ factors. We consider the Bessel beams with TE polarization in the simplest form with zero azimuthal index $m=0^{24}$

$$
\mathbf{E}_{\text {inc }}(r, \phi, z)=E_{0} \mathbf{e}_{\phi} \exp \left( \pm i k_{z} z\right) J_{1}\left(k_{r} r\right)
$$

where $J_{1}$ is an Bessel function, $k_{z}$ and $k_{r}$ are the longitudinal and transverse wave numbers, with the frequency $\omega / c=k=\sqrt{k_{r}^{2}+k_{z}^{2}}$ and $r, \phi$, and $z$ are the cylindrical coordinates, $\mathbf{e}_{\phi}$ is the unit vector of the polarization. The total system of two disks and applied Bessel beams preserves the axial symmetry that allows us to consider the simplest case with zero azimuthal index $m=0$. In order to stabilize both disks in z-direction we use the approach in which two counter-propagating mutually incoherent Bessel beams were applied 3.5 that is schematically shown in Fig. 11

At first, we considered a stability of single disk at $r=$ 0 . Numerical calculations of difference between force produced by the centered Bessel beam and the force produced by slightly shifted beam show that the Bessel beams strongly trap spherical particles at the symmetry axis, i.e., at $r=0$ (stable zero-force points) similar to the case of sphere $\frac{25}{}$. That considerably simplifies the further calculation of the $\mathrm{OB}$ force between two disks and allows to consider the optical forces over the axis of symmetry only. We define the $\mathrm{OB}$ force $F_{O B}=\left(F_{1 z}-F_{2 z}\right) / 2$ where the indices 1 and 2 note the disks where the Bessel beam incident at the left. Owing to an incoherence of the Bessel beam illuminated from the right we have the same expression for $F_{O B}=-F_{O B}^{\leftarrow}$. As a result we obtain doubled value for the OB force $F_{O B}=F_{1 z}-F_{2 z}$ and zero optical pressure on both disks. From Fig. 3 one can see that the $\mathrm{OB}$ force is sensitive to the resonant frequencies shown by green solid (bonding) and dash (anti-bonding) lines. One can that the bonding and anti-bonding resonances shown in Fig. 2 which achieve the $Q$ factor above 400 have no significant bright effect effect on the OB force as Fig. 3 shows. We see that maximal $\mathrm{OB}$ force is enhanced by three orders and reached a value up to one femto Newton. 


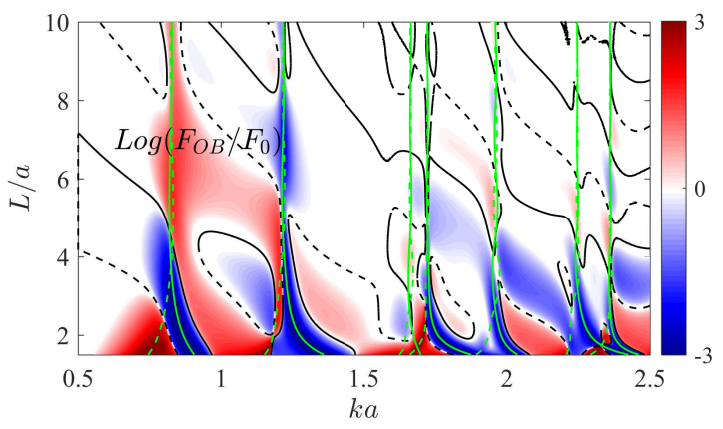

FIG. 3. The OB force between two disks vs the frequency and distance between centers of disks with radius $a=0.5 \mu \mathrm{m}$, aspect ratio $a / h=0.71$ and $\varepsilon=12$ under illumination of the Bessel beam with TE polarization and $k_{z} a=1$. Black solid (dash) lines show stable (unstable) configuration of disks. Green solid (dash) lines show anti-bonding (anti symmetric) and bonding (symmetric) resonant frequencies of two disks vs the distance between. $F_{0}=1 p N$.

Next, we show in Fig. 2] (a) the antisymmetric resonance (black dash line) crosses the symmetric resonance (red solid line) at $a / h=1.009$. These resonances are not coupled in the isolated disk because of their orthogonality to each other. However as soon as the second disk is approaching this symmetry prohibition is lifted. Evolution of these resonances with distance $L$ between disks is shown in Fig. 4 When the distance is large enough the resonances marked by green crosses are degenerate. Let define the corresponding modes as $\psi_{1}(\vec{r})$ and $\psi_{2}(\vec{r})$ which are shown in Fig. 4 in upper insets. With approaching of disks these resonant modes are hybridizing as follows 23

$$
\psi_{1,2 ; s, a}(\vec{r}) \approx \psi_{1,2}\left(\vec{r}_{\perp}, z-\frac{1}{2} L \vec{z}\right) \pm \psi_{1,2}\left(\vec{r}_{\perp}, z+\frac{1}{2} L \vec{z}\right)
$$

where $\vec{z}$ is the unit vector along the z-axis. These modes can be classified as bonding (symmetrical) or anti-bonding (anti-symmetrical) resonant modes and illustrated in Fig. 4 at $L=5 a$. However with further approaching of disks the approximation (2) ceases to be correct because of interaction of the resonances $\psi_{1}$ and $\psi_{2}$. One can observe noticeable deformation of these resonant modes at $L=2.5 a$ in Fig. 4 and especially at $L=1.75 a$. At this distance and the aspect ratio $a / h=1.009$ the anti-bonding resonant mode highlighted in Fig. 4 by open circle is featured by extremal low resonant width as marked by rhombus. Respectively, one can observe extremely high peak of the $Q$ factor around 5500 in Fig. 5] at the vicinity $L=1.75 a$ and $a / h=1.009$. The further approaching of disks until they stick each other at $L=a$ results in the resonant modes surprisingly close to approximation (2) as insets at the right of Fig. 4 4 show. The reason of extremely small radiation losses of the anti-bonding mode at $L=1.75 a$ and $a / h=1.009$ is related to its morphology which is immensely close to the morphology of the Mie resonant mode with orbital momentum $l=6$ of a sphere with volume $\pi(h+L) a^{2}$. That sphere is highlighted in the corresponding inset in Fig. 4 by open white circle.

It is worthy to note that this case of extremal enhancement

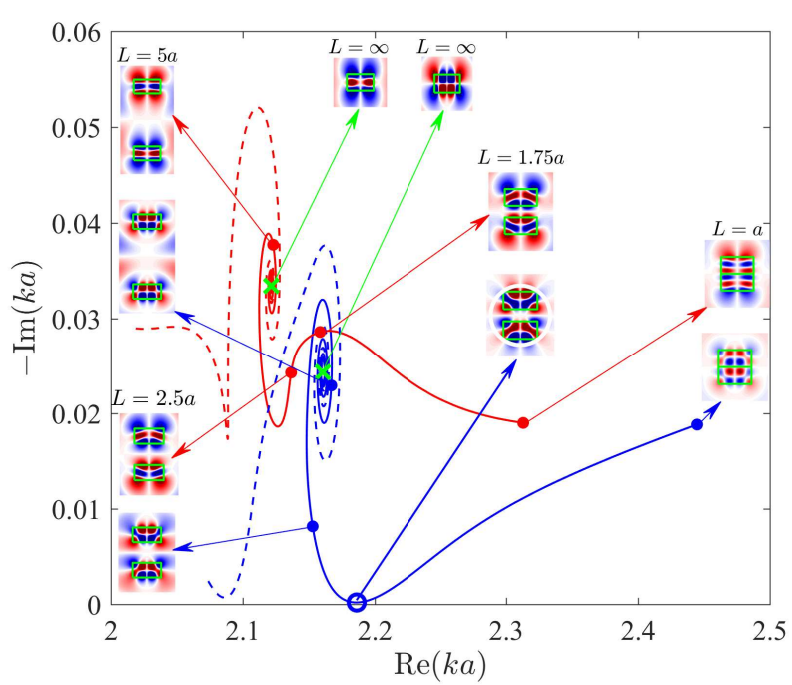

FIG. 4. Avoided crossing of resonances originated from coupling of orthogonal resonances of isolated disk shown in Fig. 2 (a) for variation of distance between disks for $h / a=1.009$. Solid/dash lines show the anti-bonding/bonding resonances. Insets show the profiles of tangential component of electric field $E_{\phi}$.

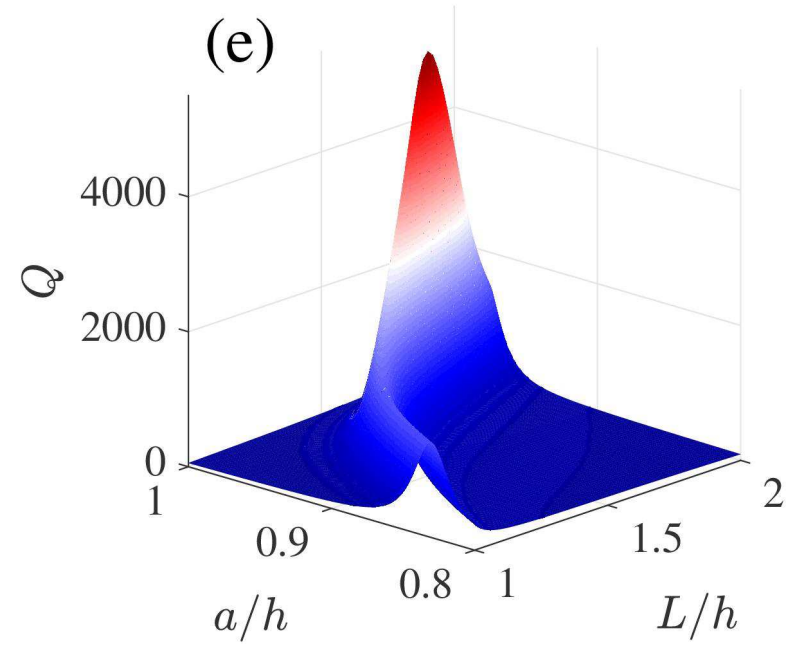

FIG. 5. The $Q$ factor vs aspect ratio and distance between disks of the anti-bonding resonant mode (blue line in Fig. 4 highlighted by open circle).

of the $Q$ factor due to avoided crossing of orthogonal resonant modes of isolated disk is not unique. Fig. 6, for example, demonstrates another scenario of the avoided crossing for approaching of disks with aspect ratio $a / h=1.17$ however for higher lying resonant modes. The right inset shows that the anti-bonding resonant mode which demonstrates unprecedented $Q$ factor 15000 at $L=1.4 a$. Similar to the case shown Fig. 4the reason of that is the morphology of the anti-bonding resonant mode is close to the Mie resonant mode with $l=8$.

Fig. 7 shows general picture for the OB force at $a / h=$ 1.009 in Log scale versus the frequency of the dual Bessel 


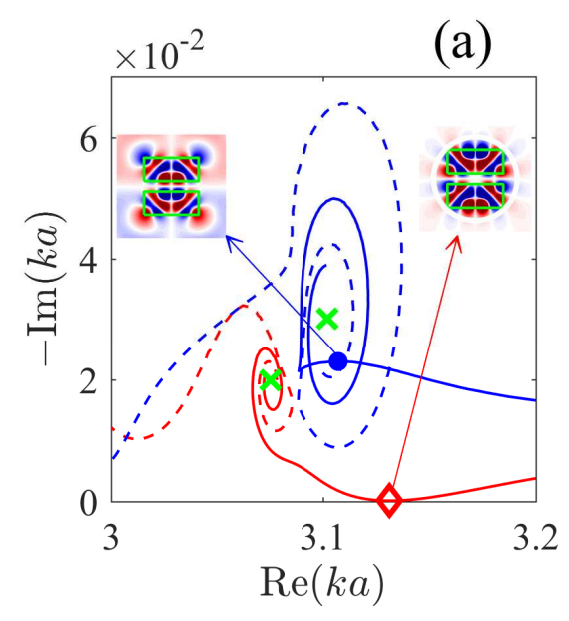

(b)

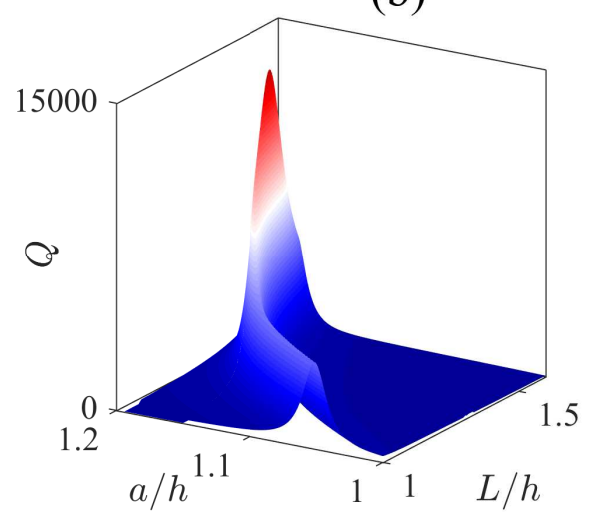

FIG. 6. (a) Evolution of the higher lying TE resonances in traversing with the distance between the disks for $a / h=1.17$. Solid/dash lines show the anti-bonding/bonding resonances. (b) The $Q$ factor vs the distance between the disks and their aspect ratio.

beams and distance $L$. In order the reader could imagine the extremal behavior of the OB force we reproduce fragment of Fig. 7 as surface in Fig. 8 (a) where one can see that giant $\mathrm{OB}$ is achieved around 30 femto Newtons at $k a=1.97, L=$ $1.85 a, h=1.03 a, k_{z} a=0.5$. Fig. 8 (b) shows that this giant peak is split for $k_{z} a=1$. It is remarkable that the equilibrium distances between disks is traversed close to the anti-bonding resonance shown by solid line. Fig. 9 demonstrates as this giant peaks in $\mathrm{OB}$ are easily manipulated by small changes in

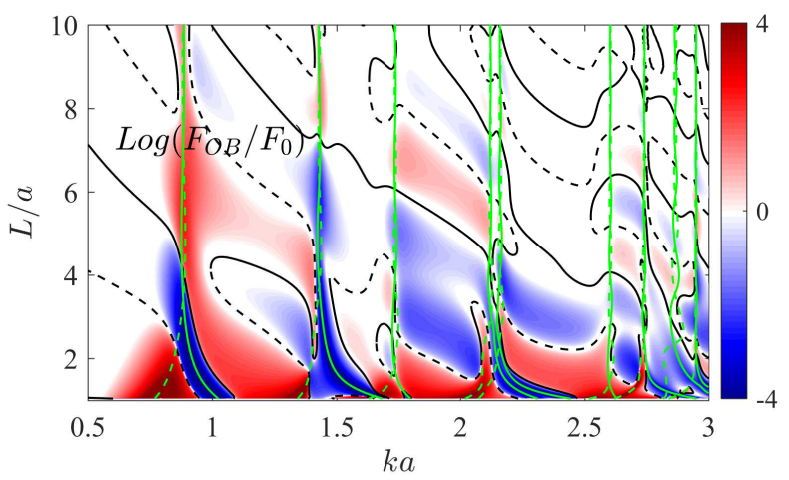

FIG. 7. The binding force between two disks vs the frequency and distance between centers of disks with aspect ratio $a / h=1.0395$ for the Bessel beam with TE polarization $k_{z} a=1 / 2$ where the disk with $\varepsilon=12$ has the radius $a=0.5 \mu \mathrm{m}$.
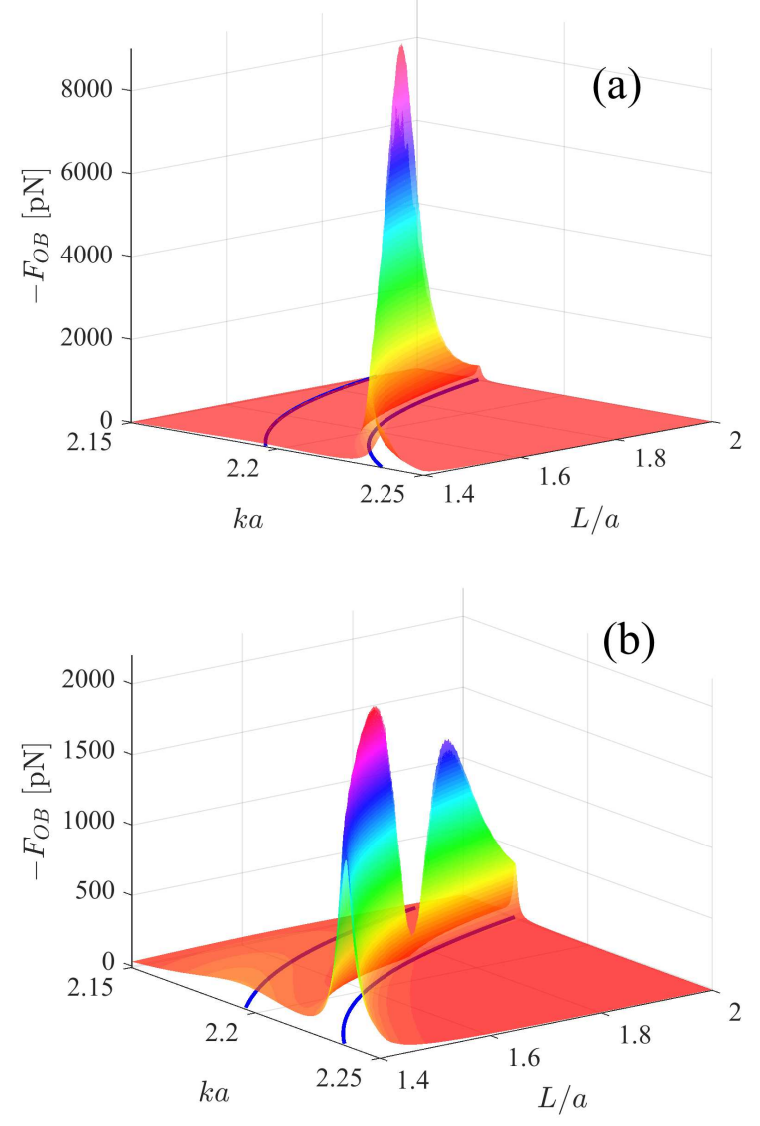

FIG. 8. The OB vs distance between centers of disks at the vicinity of the anti-bonding resonance marked in Fig. 2 (c) by closed circle $k a=1.95$ (a) $k_{z} a=0.5$ and (b) $k_{z} a=1$. Solid line underneath shows anti-bonding resonant frequencies vs distance $L$ highlighted in Fig. 5 

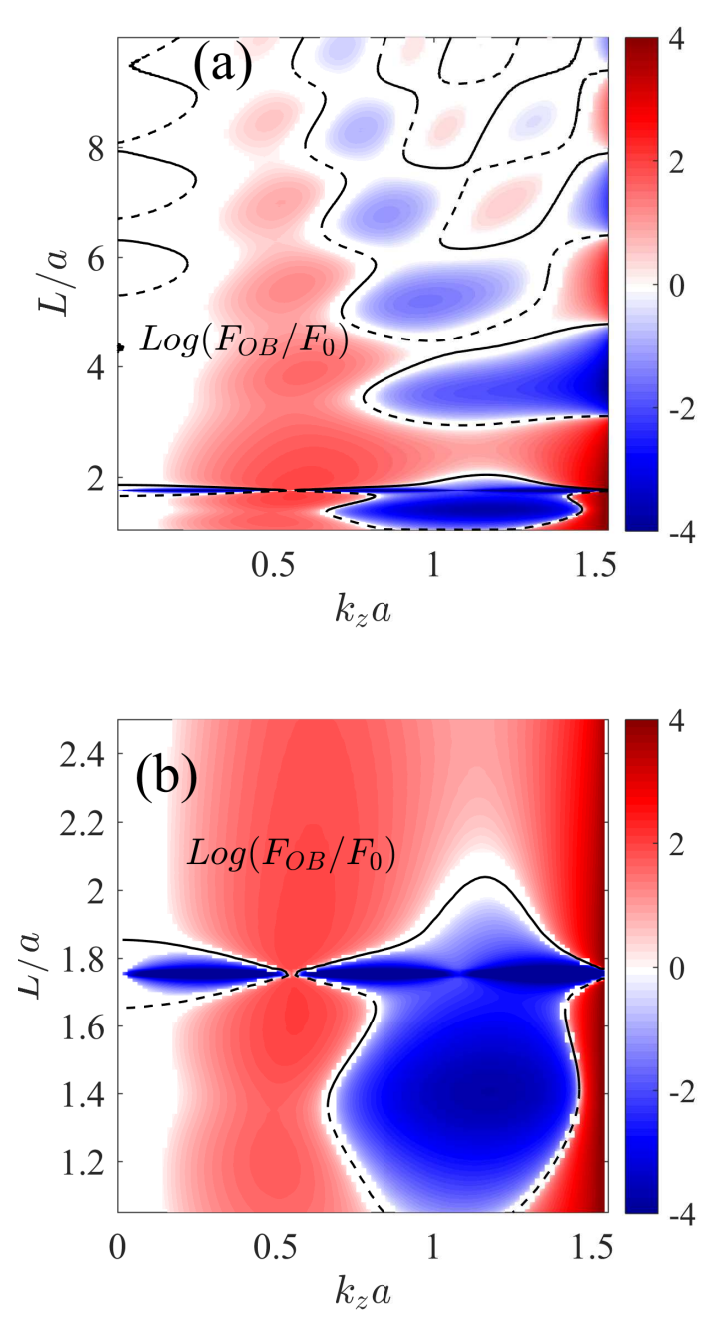

FIG. 9. (a) The OB vs distance between disks and longitudinal wave vector of the Bessel beam $k_{z} a$ at the vicinity of the anti-bonding resonance marked in Fig. 2 (c) by closed circle $k a=1.95$. (b) Zoomed version of (a).

parameters of the Bessel beam: $k_{z} a$ and frequency.

\section{SUMMARY}

In the present paper we considered the resonant enhancement of the OB force of two silicon disks of micron size by illumination of dual incoherent counter propagating Bessel beams. As distinct from the case of two dielectric spheres 10,11 the case of coaxial disks brings new aspect for the OB force related to the extremely high $Q$ factor due to two-parametric avoided crossing of orthogonal resonances over aspect ratio and distance between the disks ${ }^{19}$. The corresponding antibonding resonant modes of two disks are turned out to be closed to the Mie resonant mode with high orbital index $l=6$ or even $l=8$ of an effective sphere with volume $4 \pi R^{3} / 3=$ $\pi(h+L) a^{219}$ that explains the extremely high $Q$ factors. For the case of two coaxial silicon disks with micron diameter illuminated by dual coaxial Bessel beams we demonstrate giant OB force in few decades of femto Newtons in a vicinity of anti-bonding resonances. Giant enhancement of optical forces have been reported already $13,14,16$ however for PhC layers. It is remarkable that the OB force can be easily manipulate by the counter propagating Bessel beams, by tuning of the frequency onto the the resonances of anti-bonding resonances and by width of the Bessel beams.

\section{Acknowledgments}

The work was supported by Russian Foundation for Basic Research projects No. 19-02-00055.

${ }^{1}$ A. Ashkin, J. M. Dziedzic, J. E. Bjorkholm, and S. Chu, "Observation of a single-beam gradient force dielectric particles," Opt. Lett. 11, 290-292 (1986).

${ }^{2}$ M. M. Burns, J.-M. Fournier, and J. A. Golovchenko, "Optical binding," Phys. Rev. Lett. 63, 1233 (1989).

${ }^{3}$ S. A. Tatarkova, A. E. Carruthers, and K. Dholakia, "One-dimensional optically bound arrays of microscopic particles," Phys. Rev. Lett. bf 89, 283901 (2002).

${ }^{4}$ R. Gomez-Medina and J. J. Sáenz, "Unusually strong optical interactions between particles in quasi one-dimensional geometries," Phys. Rev. Lett. 93, 243602 (2004).

${ }^{5}$ N. K. Metzger, K. Dholakia, and E. M. Wright, "Observation of bistability and hysteresis in optical binding of two dielectric spheres," Phys. Rev. Lett. 96, 068102 (2006).

${ }^{6}$ N. K. Metzger, E. M. Wright, and K. Dholakia, "Theory and simulation of the bistable behaviour of optically bound particles in the Mie size regime," New J. Phys. 8, 139 (2006).

${ }^{7}$ K. Dholakia and P. Zemánek, "Colloquium: Gripped by light: Optical binding," Rev. Mod. Phys. 82, 1767 (2010).

${ }^{8}$ R. Bowman and M. Padgett, "Optical trapping and binding," Rep. Progr. Phys. 76, 026401 (2013).

${ }^{9}$ I. Thanopulos, D. Luckhaus, and R. Signorell, "Modeling of optical binding of submicron aerosol particles in counterpropagating bessel beams," Phys. Rev. A 95, 063813 (2017).

${ }^{10} \mathrm{P}$. C. Chaumet and M. Nieto-Vesperinas, "Optical binding of particles with or without the presence of a flat dielectric surface," Phys. Rev. B 64, 035422 (2001).

${ }^{11} \mathrm{~J} . \mathrm{Ng}, \mathrm{Z}$. Lin, C. Chan, and P. Sheng, "Photonic clusters formed by dielectric microspheres: Numerical simulations," Phys. Rev. B 72, 085130 (2005).

${ }^{12} \mathrm{~T}$. Čižmár and V. Kollárová and Z. Bouchal and P. Zem'anek, "Sub-micron particle organization by self-imaging of non-diffracting beams," New J. Phys. 8, 43 (2006).

${ }^{13}$ M. I. Antonoyiannakis and J. B. Pendry, "Mie resonances and bonding in photonic crystals," Europhys. Lett. 40, 613 (1997).

${ }^{14}$ V. Liu, M. Povinelli, and S. Fan, "Resonance-enhanced optical forces between coupled photonic crystal slabs," Opt. Express 17, 21897 (2009).

${ }^{15}$ A. Rodriguez, A. McCauley, P.-C. Hui, D. Woolf, E. Iwase, F. Capasso, M. Loncar, and S. Johnson, "Bonding, antibonding and tunable optical forces in asymmetric membranes," Opt. Express 19, 2225 (2011).

${ }^{16}$ J. Zhang, K. MacDonald, and N. Zheludev, "Giant optical forces in planar dielectric photonic metamaterials," Opt. Lett. 39, 4883 (2014).

${ }^{17}$ M. Benyoucef, J.-B. Shim, J. Wiersig, and O. G. Schmidt, "Quality- factor enhancement of supermodes in coupled microdisks," Opt. Lett. 36, 1317 (2011).

${ }^{18}$ M. L. Povinelli, S. Johnson, M. Loncar, M. Ibanescu, E. Smythe, F. Capasso, and J. D. Joannopoulos, "High-Q enhancement of attrac- tive and repulsive optical forces between coupled whispering-gallery- mode resonators," Optics Express bf 13, 8286 (2005).

${ }^{19}$ E. N. Bulgakov, K. N. Pichugin, and A. F. Sadreev, "Engineering of the extremely high-Q factor in two subwavelength dielectric resonators," arXiv:2005.05554 1 .

${ }^{20} \mathrm{~J}$. Wiersig, "Formation of Long-Lived, Scarlike Modes near Avoided Resonance Crossings in Optical Microcavities," Phys. Rev. Lett. bf 97, 253901 (2006). 
${ }^{21} \mathrm{~S}$. V. Boriskina, "Theoretical prediction of a dramatic Q-factor enhancement and degeneracy removal of whispering gallery modes in symmetrical photonic molecules," Opt. Lett. 31, 338 (2006).

${ }^{22}$ M. Rybin, K. Koshelev, Z. Sadrieva, K. Samusev, A. Bogdanov, M. Limonov, and Y. Kivshar, "High-Q Supercavity Modes in Subwavelength Dielectric Resonators," Phys. Rev. Lett. bf 119, 243901 (2017).
${ }^{23}$ K. N. Pichugin and A. F. Sadreev, "Interaction between coaxial dielectric disks enhances the $Q$ factor," J. Appl. Phys. 126, 093105 (2019).

${ }^{24} \mathrm{~V}$ Karásek and O Brzobohatý and P Zemánek, "Longitudinal optical binding of several spherical particles studied by the coupled dipole method," J. Optics A: Pure and Appl. Optics 11, 034009 (2009).

${ }^{25}$ G. Milne, K. Dholakia, D. McGloin, K. Volke-Sepulveda, and P. Zemánek, "Transverse particle dynamics in a bessel beam," Opt. Express 15, 13972 (2007). 